\title{
El tamaño de la empresa y su influencia en la productividad del sector comercio
}

\section{Firm size and its influence on productivity in the commerce sector}

Alexander Fernando Haro Sarango

Universidad Técnica de Ambato, Ambato, Ecuador

aharo8014@uta.edu.ec

D https://orcid.org/0000-0001-7398-2760

Recepción: 13/06/2021 | Aceptación: 24/08/2021 | Publicación: 10/09/2021

Cómo citar (APA, séptima edición):

Haro Sarango, A. F. (2021). El tamaño de la empresa y su influencia en la productividad del sector comercio. INNOVA Research Journal, 6(3), 227-245.

https://doi.org/10.33890/innova.v6.n3.2021.1781

\section{Resumen}

En el entorno investigativo empresarial existe una discusión constante sobre sí, el tamaño de la empresa es un elemento circunstancial para la supervivencia económica, conjuntamente, sobre los riesgos existentes y los factores que pueden generar problemas en una organización; este estudio pretende indagar sobre la influencia del tamaño de la empresa en la productividad perpetuada, la temporalidad radica en el periodo 2016 al 2020, CIIU "G"; los sujetos de estudio para esta investigación son: 15\% (139) empresas grandes, 39\% (361) medianas y 47\% (445) pequeñas; los indicadores a usar son: índice Dupont, margen de rentabilidad neta sobre ventas, rotación de activos, multiplicador de capital, costo de financiamiento y operativos, la metodología para conocer la influencia de las variables usadas serán los modelos efectos fijos y aleatorios, previa consideración de Hausman, asimismo, una correlación con la finalidad de encontrar una relación entre variables directa o inversamente proporcional; por otro lado, para discernir sobre la influencia del tamaño de las empresas en los estratificaciones usadas se consentirá mediante ANOVA para grupos y Turkey para secciones. Los resultados del estudio determinan que el costo financiero y operativo no son variables significativas en la productividad, por otro lado, existe una alta afinidad en la gestión de las empresas grandes y medianas, adicional a esto, las empresas pequeñas presentan menor costo financiero y operativo y un mayor índice Dupont, con lo cual se determina que existe una ligera diferencia en algunas variables con respecto al tamaño de la empresa

Palabras claves: tamaño de la empresa; productividad; Dupont; capital. 


\begin{abstract}
In the business research environment there is a constant discussion about whether, the size of the company is a circumstantial element for economic survival, jointly, about the existing risks and factors that can generate problems in an organization; this study aims to inquire about the influence of the size of the company on the perpetuated productivity, the temporality lies in the period 2016 to 2020, ISIC "G"; the subjects of study for this research are: 15\% (139) large companies, 39\% (361) medium and 47\% (445) small; the indicators to be used are: Dupont index, net profitability margin over sales, asset turnover, capital multiplier, financing and operating cost, the methodology to know the influence of the variables used will be the fixed and random effects models, prior consideration of Hausman, also, a correlation with the purpose of finding a relationship between variables directly or inversely proportional; on the other hand, to discern about the influence of the size of the companies in the stratifications used will be consented through ANOVA for groups and Turkey for sections. The results of the study determine that the financial and operating cost are not significant variables in productivity, on the other hand, there is a high affinity in the management of large and medium-sized companies, in addition to this, small companies present lower financial and operating cost and a higher Dupont index, which determines that there is a slight difference in some variables with respect to the size of the company.
\end{abstract}

Keywords: company size; productivity; Dupont; capital.

\title{
Introducción
}

En entornos volátiles y con crisis, el tamaño de la empresa se ejemplifica como el factor primordial de la supervivencia y crecimiento organizacional, si bien se sabe las pymes se consideran el tejido económico-productivo de una economía, presenta un significativo nivel de fragmentación y quiebre debido a su reducido tamaño y concentración de capital (Prats y Merino, 2015). Antagónicamente, Vilabella y Silvosa (2001) asevera que no existe diferencias abismales entre estos pragmáticos grupos empresariales en el ámbito financiero, asimismo confirma que la generalidad del riesgo aún sigue existiendo, debido a que las empresas pequeñas inician con alto nivel de endeudamiento a corto plazo, lo cual es una desventaja en el contexto del circulante. En la conceptualización de la innovación, Camison et al., (2002) alude que la relación entre la variable tamaño-innovación tiene alta afinidad e influencia, debido a la falta de adherencia a la investigación y desarrollo (I+D), así como a la falta de recursos asignados a dicha índole.

Las empresas grandes y con mayor cantidad de recursos tienen una organización compleja y de difícil tratamiento (Garrido y Peres Núñez, 1998). En la gestión administrativa, específicamente en la evaluación y auditoría, el tamaño de la empresa influye en la calidad de la auditoria, lo cual indica que por su dimensión puede estar inmersa la posibilidad de cometer errores (Aguiar-Díaz y Díaz-Díaz, 2015), actualmente el proceso de evaluación y las nuevas normas se han adaptado de mejor forma a la era tecnológica, las Normas Internacionales de Auditoría (NIAs) como estándares de medición, las métricas de gestión y los sistemas contables, funcionan en conjunto con la tecnología de la información lo cual no limita el apoyo a la gestión auditora y permite tener toda la organización a la mano (De la Peña Gutiérrez, 2014; Luna, 2015; Martínez et al., 2012; Pelazas, 2015), aunque el mundo avance a gran velocidad, la adaptabilidad es la herramienta apropiada para superar nuevos retos. 
El objetivo de la investigación es determinar, contextualizar y discernir sí el tamaño de la empresa influye en la productividad, asimismo, se pretende indagar en la significancia de determinadas variables afín al costo por apalancamiento y operatividad, con la finalidad de verificar si la administración de estas cuentas está ligada a la productividad, debido a que siempre se categoriza a las empresas que no son grandes como más riesgosas, aunque la afirmación es parcialmente correcta, es circunstancial complementar que, no porque su adherencia al riesgo sea más alta no pueda ser productiva y rentable, por lo cual se evaluará mediante el argumento de productividad a las empresas del sector comercio, la misma que según La Clasificación Industrial Internacional Uniforme (CIIU) poseen el código "G46 - comercio al por mayor, excepto el de vehículos automotores y motocicletas" (A. García, 1981).

\section{Marco teórico}

La era tecnológica ha concebido un sinnúmero de herramientas que han logrado mitigar las consecuencias de las crisis económicas y financieras. En el entorno empresarial existen variedad de mecanismos con los cuales se diagnostica escenarios, las mismas facultan a la estructuración de soluciones inmediatas (Herrera, 2012). Con respecto a la historia, el físico matemático Edwards Deming alude que los principios organizacionales fundados en la filosofía condescendería entender cómo funcionan las cosas, basado en la calidad en una organización (Deming y Medina, 1989); el profesor japonés Kaouro Ishikawa ratifica en una nueva filosofía basada en el control de la calidad total, el cual es un esquema que permite a la administración direccionar esfuerzos a la mejora, asimismo, menciona que la responsabilidad recae en todos los miembros de la organización como un grupo, el cual debe orientarse a la calidad (Ishikawa, 2003). Pero que tiene que ver la calidad con la productividad, Gutiérrez Pulido (2010) asevera que, el mundo es cada día más globalizado, por lo cual las exigencias para las organizaciones son más rígidas, por dicha razón, el vínculo entre la calidad y la productividad son cada vez más significativos, mediante la gestión de la calidad se logrará una organización con manufactura esbelta, por ende, el nivel de productividad incrementará, la relación intrínseca entre estas variables posibilita la mejora de la competitividad y evita el desperdicio de esfuerzos.

\section{La era tecnológica: datos masivos e inversión en TIC y su relación con la productividad}

La terminología de productividad tiene un amplio campo de estudio y relaciones, cada autor asevera que existen una infinita gama de variables que pueden incidir en dichos indicadores, no obstante, cada cualidad permite crear diferentes hipótesis que solventan la gestión eficaz.

Torrecilla (2005) manifiesta que la productividad reside principalmente en la satisfacción del recurso humano, confiere que los modelos organizacionales buscan esquemas de motivación que permitan generar un clima favorable para su propicio desempeño. El umbral de un nuevo mundo y el desarrollo de las Tecnologías de la Información y de las Comunicaciones (TIC), concede que la inversión en este ámbito favorece a los indicadores de productividad, una muestra realizada a 500 empresas, determinan que por cada dólar asignado en capital TIC, se produce un incremento de 0,81 y de 2,62 dólares en el producto de la organización (Brynjolfsson y Yang, 1996; Dewan y Kraemer, 2000; Sánchez, 2004). El uso de datos masivos gestionados para la toma de decisiones, Big Data ha traspasado las fronteras, hablando de perspectivas de desarrollo local e internacional, las inversiones direccionadas a las dependencias de capacidad, almacenamiento y 
procesamiento para la exploración y generación de datos, permiten a una organización tener una infraestructura de redes de alta velocidad e innovación, Mckinsey Global Institute (MGI) argumenta que las herramientas Big Data convierte los datos en factores de producción solventado una ventaja competitiva, sugiere que las empresa del futuro deberán adaptarse al mundo de los datos (Aguilar, 2016; Malvicino y Yoguel, 2017; Tascón, 2013).

Como se mencionó con anterioridad la productividad tiene diferentes vínculos con múltiples aspectos, sin embargo, es necesario aclarar que cuando se origina un estudio la estratificación es circunstancial para determinar las variables significativas, la cualidad de segmentar es oportuno para abordar un análisis entre sujetos con las mismas particularidades.

\section{Enfoque teórico de la administración y finanzas en la productividad}

La mejora continua representa un reto para las empresas, la optimización de los factores productivos y los recursos conlleva un análisis complejo para alcanzar el éxito empresarial, por lo cual, el PHVA (Planificar, Hacer, Verificar y Actuar) surge como una herramienta para resolver problemas, por ende, mejorar el desempeño organizacional, basado en un sistema justo a tiempo, control de calidad y políticas eficaces que se formulan entorno a los fundamentos de mejora continua, se ha demostrado que esta metodología forja ideales de desarrollo competitivo en un sinnúmero de entidades, por lo cual su efectividad ha sido justificada con demasié (Cuadros y Angel, 2019; Zapata, 2016).

Tárraga (2011) argumenta sobre las Practicas de Alta Implicación o Rendimiento (del inglés HIWP), grupo de destrezas de la gestión del talento humano que busca la mejora organizacional mediante la sinergia, su estudio determina que estas prácticas mejoran el performance de las empresas y modernizan los enfoques y estrategias, por ende, desarrolla la productividad.

En el marco de la gestión, el Cuadro de Mando integral o Balanced Scorecard, se posiciona como un instrumento de gestión estratégica que confiere esquemas de comunicación eficiente, desarrollan la objetividad lo cual brinda una disminución de los tiempos, su aplicación como herramienta gerencial transforma a las empresas y las vuelve significativamente productivas (Scaramussa et al., 2010).

En el campo financiero, específicamente en las fuentes de financiación podemos acotar que existen diversidad de productos financieros, sin embargo, no todos son propicios para las empresas, debido al Costo Anual Total (CAT), que se refleja en la carga financiera y los periodos de pago que se descuadran de las fechas de recuperación de la cartera, no obstante, al existir variedad como se mencionó, es tarea de la administración evaluar la viabilidad de la fuente de financiación con terceros para solventar el potencial de determinados recursos en la operabilidad.

Las diversas teorías ratifican que el comportamiento financiero en base a la solvencia y rentabilidad, aseguran a una entidad a ser económicamente viable y sostenible a largo plazo; los indicadores de liquidez, endeudamiento, gestión y rentabilidad actúan como las métricas propicias para evaluar el comportamiento de la entidad y diagnosticar a tiempo, con la finalidad de corregir el camino de la empresa y evitar la adherencia al riesgo (Acín, 1996; Altman, 1968). 
El término generación de valor es muy usado en la gestión empresarial, promete una evaluación que condesciende observar si una empresa es productiva y rentable, aunque muchos lo categorizan como el beneficio que obtiene una organización al final de un periodo, otros como la eficacia con la que hace frente a las obligaciones financieras, asimismo algunos, lo califican como la cuantía direccionada a la reinversión (Correa-García et al., 2018); sin embargo, esta cualidad está ligada a las estrategias de optimización productiva, es decir a la detección inmediata de falencias y la evaluación de viabilidad-sostenibilidad; una de las metodologías conocidas para medir la generación de valor es mediante el EVA (Economic Value Added) (Rivas Martínez y Montero Cabas, 2020).

\section{Factores determinantes en el tamaño de la empresa, estructura y productividad}

Los estudios empíricos sobre la combinación de recursos propios y ajenos para unificar un valor considerable y sustancial para la operatividad abundan, cada uno de ellos presentan contextos diferentes; Jiménez Naharro y Palacín Sánchez (2007) asevera que, la mayoría de empresas presentan una carga significativa de endeudamiento, alrededor del 68,41\% al 72,05\%, sin embargo, aquí es necesario conocer sí esta carga excesiva de cuentas con terceros perjudican al desarrollo propicio de la entidad, Haro (2021) argumenta en un planteamiento basado en riesgo y rentabilidad que una empresa con un valor de endeudamiento entre el 50 al $70 \%$ es segura, siempre y cuando los recursos tengan una administración coherente afín a la solvencia y reinversión.

Adicional a esto, es circunstancial evaluar qué tan significativo es el costo financiero con relación a los productos económicos adquiridos. Sanz y Ayca (2006) aluden que el costo financiero es uno de los principales problemas de insolvencia en las empresas Latinoamericanas, debido a su alto valor que es imposible cubrir con la operatividad, por otro lado, argumentan que si no existe esa captación monetaria es complicado acceder a un mercado competitivo; Colina (2006) ratifica que no solo se debe considerar los costos financieros, sino, debe existir una inclusión de todas las fuentes de costos, con la premisa de inducir a un diseño estratégico conducido a la satisfacción por medio del rendimiento. En congruencia con lo anterior, Cabellos Mendo y Naito Endo (2015) asegura que el tratamiento del índice Dupont (productividad) tiene alta relación con el costo de la deuda y el apalancamiento financiero, asegura que su relación es significativa.

Terreno (2013) en su investigación determina que existe una alta afinidad entre el tamaño empresarial afín a la estructura patrimonial y los derivados de los costos, asimismo, menciona que estas variables son significativas con respecto a los valores de utilidad y productividad. Por último, Zabaleta García (2012) infiere que la generación de valor y productividad está ínfimamente vinculado al alza contante de las variaciones de capital y valores monetarios

\section{Metodología}

\section{Población y estratificación}

Para este estudio se comienza con la estratificación del tamaño de las empresas, según la SUPERCIAS (2020) su cualificación es la siguiente: 


\section{Tabla 1}

Cuartiles de los indicadores de productividad

\begin{tabular}{ll}
\hline Tamaño & Nivel de ingresos \\
\hline Empresa Pequeña & $\$ 100.001,00$ y $\$ 1^{\prime} 000.000,00$ \\
Mediana Empresa & $\$ 1^{\prime} 000.001,00$ y $\$ 5^{\prime} 000.000,00$ \\
Empresa Grande & Superiores a los $\$ 5^{\prime} 000.001,00$
\end{tabular}

Nota: Estratificación realizada por la Superintendencia de Compañías Valores y Seguros

Por otra parte, la segmentación por actividad económica generada para este estudio según La Clasificación Industrial Internacional Uniforme (CIIU) es "G46 - comercio al por mayor, excepto el de vehículos automotores y motocicletas".

El estudio tendrá la temporalidad de 5 años, comprendidos desde el 2016 hasta el 2020, su desarrollo territorial comprende a las empresas que residen en Ecuador que se encuentren legalmente registradas en la Superintendencia de Compañías Valores y Seguros, por otra parte, como un medio de evaluación de los estados financieros se observará mediante la fórmula contable si los valores son acordes y no existe errores de registro, esto se realiza para evitar cifras que perjudiquen la validez del estadístico. Asimismo, el estudio no tendrá muestra, se trabajará con la población total de empresas, con dicho esquema se pretende aumentar el nivel de confianza de los resultados. La población de estudio resultante de la evaluación previa y estratificación es la siguiente:

\section{Figura 1}

\section{Población de estudio}

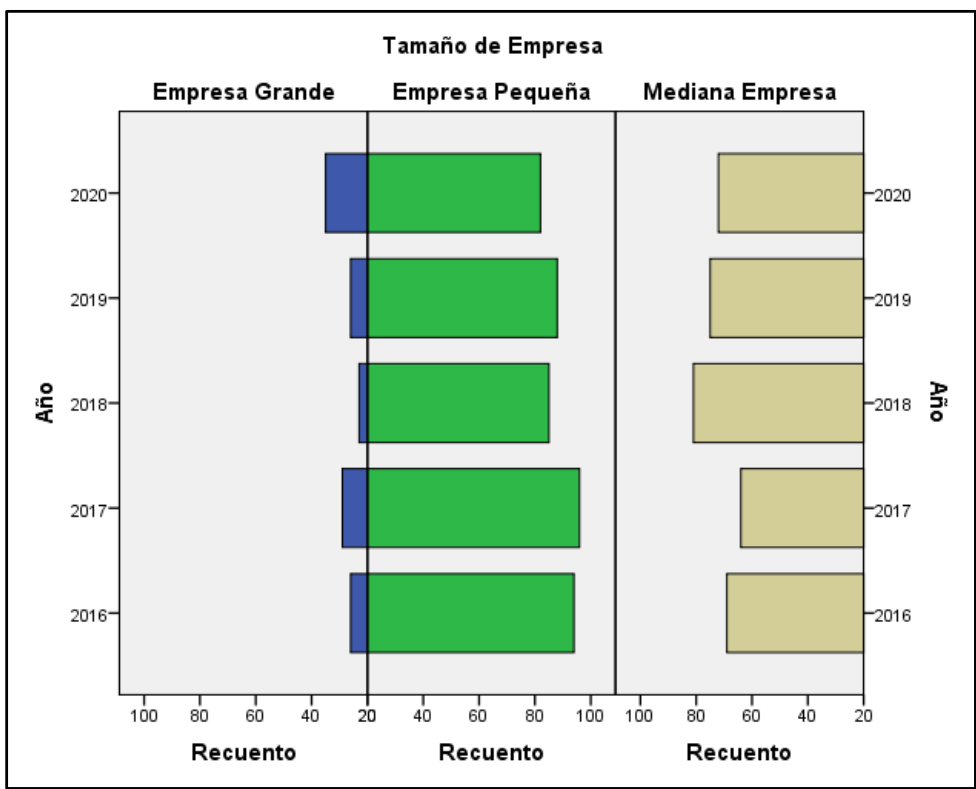

Fuente: Elaboración propia, mediante la representación gráfica en el aplicativo SPSS v.21 
La población total analizada es de 945 empresas, distribuidas de forma uniforme en cada año; las empresas grandes representan el 15\% (139), las medianas el 38\% (361) y las pequeñas el $47 \%$ (445); como se observa en la figura 1. la distribución del tamaño de las empresas por cada periodo evaluado es uniforme, y su varianza es mínima.

\section{Tipo de investigación, enfoque y tratamiento de variables}

El enfoque metodológico de la investigación es de carácter cuantitativo, debido al manejo de indicadores financieros que presentan el carácter medible; el tipo de estudio es correlacional, generado por la comparación entre variables y la determinación de la influencia y congruencia entre las mismas; basado en la representación numérica de las variables, estás toman la característica de continuas, porque se distribuyen dentro de intervalos y su representación es libre de caracteres.

\section{Métricas de evaluación de la productividad}

\section{Índice Dupont}

El encuadre del modelo simplifica el análisis financiero ejemplificando una relación entre valores del potencial de una entidad para forjar rentabilidad, el uso de recursos para el giro del negocio y el financiamiento propio; mediante la formulación de la ecuación unificada por el múltiplo de los tres elementos se observará las causas intrínsecas que generan la rentabilidad en la empresa (O. L. García, 2009). La ecuación del índice Dupont según el autor citado es la siguiente:

\section{$\frac{\text { Utilidad neta }}{\text { Ventas }} x \frac{\text { Ventas }}{\text { Activos fijos }} x \frac{\text { Activo total }}{\text { Patrimonio }}$}

Los elementos desagregados del índice Dupont se detallan a continuación:

- Margen de rentabilidad neta en ventas: la utilidad neta es el valor resultante de la operación lucrativa de la empresa descontado los costos, gastos, intereses e impuestos generados en la actividad económica (Gitman, 2003); la rentabilidad financiera es una cuantificación relativa que busca comparar la utilidad frente a las ventas, este indicador faculta a la empresa a identificar que tanta capacidad tiene para ser productiva (Morillo, 2001).

\section{Utilidad neta \\ Ventas}

- Rotación de activos: considerado un indicador de eficiencia en la actividad empresarial, indica la capacidad que posee y usa una empresa para la obtención de ingresos (Rosillón, 2009).

\section{Ventas \\ Activos}


- Multiplicador de capital: denominado apalancamiento, es una consideración cuantificada de forma relativa el cual determina el grado de funcionalidad de los recursos propios (Masgrau, 2005).

\section{Activo total \\ Patrimonio}

\section{Costo del financiamiento}

Son las decisiones que toma una empresa con respecto a los flujos del efectivo para satisfacer la administración del capital de trabajo y el circulante; la costeabilidad del financiamiento radica en los intereses y la temporalidad (Díaz, 2012).

\section{Intereses pagados \\ Utilidad neta}

\section{Costos operativos}

Esta relación ejemplifica que tanto ocupa la actividad operativa en los ingresos de empresa, calculado en una temporalidad específica, en el cual se observará la administración productiva en el esquema de la costeabilidad.

\section{Costos operativos}

\section{Ingresos}

\section{Modelo de efectos fijos o aleatorios}

Siendo la distribución de la información datos de panel es propicio evaluar las variables con mayor influencia en la productividad mediante el planteamiento de hipótesis con base en el comportamiento de los residuos por reducción de suposiciones; el modelo de efectos fijos se descompone en dos partes, ya sea en una constante para los individuos o una aleatoria, esta se genera al insertar variables condicionales categóricas por los sujetos analizados, el cálculo de este es interpretado fácilmente por MCO (Granados, 2011); la formulación es la siguiente:

$$
y_{i t}=\propto+\beta X_{i t}+u_{i t}
$$

Por otro lado, el modelo de efectos aleatorios es similar y congruente al de efectos fijos, sin embargo, este añade la variable $v_{i}$, la cual afije a los sujetos de estudio en variables netamente aleatorias por causales temporales, asimismo, ejemplifica el tratamiento de los residuos a una $\operatorname{Var}\left(v_{i}\right) \neq 0$; una vez considerado esto, el modelo es el siguiente:

$$
y_{i t}=\propto+\beta X_{i t}+v_{i}+u_{i t}
$$

Por lo general el modelo de efectos aleatorios es más eficiente, debido a que la estimación de la varianza es ínfima, sin embargo, su consistencia es medianamente baja. 


\section{Prueba de especificación, Test de Hausman}

Post evaluación de los estimadores surge la incógnita, cuál estadístico es más eficiente para el tratamiento de los datos de origen; por dicha cuestión, el test de Hausman infiere en la comparación de la varianza, en otras palabras, realiza la suposición entre $\theta_{1}$ y $\theta_{2}$, si las diferencias en las estimaciones no son altas, no son sistemáticas, ni tienen un sesgo o perturbación y son consistentes, se elige el más eficiente $\theta_{2}$, en caso alterno, las diferencias son significativas y sistemáticas, se usa el estimador con mayor consistencia técnica $\theta_{1}$ (Montero, 2005; Mutl y Pfaffermayr, 2011). En otras palabras, Hausman adjudica dos criterios con respecto al P-valor:

- Sí, Prob>Chi ${ }^{2}$ es menor que 0,05 se accede a presentar la metodología de efectos fijos

- Sí, Prob>Chi ${ }^{2}$ es mayor que 0,05 se consiente la metodología de efectos aleatorios

\section{Correlación de Pearson}

Tiene como objetivo medir la fuerza o grado de asociación entre dos variables aleatorias cuantitativas que poseen una distribución normal bivariada conjunta (Restrepo \& González, 2007). Sí, es cercano a -1 (inversamente proporcional), 1 (directamente proporcional) El coeficiente se define por la siguiente ecuación:

$$
p=\frac{\operatorname{cov}(x, y)}{\sigma_{x} \sigma_{y}}-1 \leq p \leq 1
$$

\section{ANOVA (análisis de varianza para comparar múltiples medias)}

Estadístico creado en 1930 por Fisher, se lo reconoce como una herramienta básica para el estudio y análisis de uno o más factores sobre la media de una variable de tipo continua; el objetivo del estadístico es comparar si los grupos tienen o no diferencia significativa entre ellos (Girden, 1992).

Las condiciones de parametricidad clasifica la diferencia de los individuos y su estratificación en grupos, así como en categorías; pero como saber si es necesario usar el análisis de la varianza (ANOVA), esto deriva a la cantidad de grupos estudiados, en caso que el estudio tenga dos variables es recomendable el $T$ de Student, por otra parte, si las particularidades evaluadas adjudican más de dos categorías es circunstancial aplicar ANOVA (Stihle y Wold, 1989).

Para Diez et al., (2019) el estadístico denominado ANOVA analiza el $F_{\text {ratio, }}$, el cuál es el valor estudiado entre las varianzas de las medias de los grupos. En específico, si $S_{1}{ }^{2}$ es la varianza de una muestra de tamaño $n_{1}$ extraída de una población normal de varianza $\sigma_{1}{ }^{2}$ y $S_{2}{ }^{2}$ es la varianza de una muestra de tamaño $n_{2}$ forjada de una población normal de varianza $\sigma_{2}{ }^{2}$, y ambas muestras son independientes, el cociente seria: 


$$
F_{\text {ratio }}=\frac{\frac{S_{1}{ }^{2}}{\sigma_{1}{ }^{2}}}{\frac{S_{2}{ }^{2}}{\sigma_{2}{ }^{2}}}
$$

Según la investigación de Rubio-Hurtado \& Berlanga-Silvente (2012) las hipótesis para el análisis del ANOVA son:

- $H_{0}$ : Afirma que las variables analizadas no poseen diferencia entre sí

- $H_{1}$ : Afirma que existe un cierto grado de diferencia entre sí

El proceso para aceptar o rechazar las hipótesis radica en la revisión del P-valor, el valor P indica la significancia de una valoración, en términos generales este encuadre es la garantía de calidad, siempre y cuando su arbitraje sea en análisis del 95\%-99\% del nivel de confianza. Se rechaza la hipótesis nula y se concede a la alternativa cuando el $p<0,5$; por otra parte, si el $p>0,05$ aceptamos la hipótesis nula donde adjudica que no hay diferencia explicativa.

\section{Prueba de Tukey}

García-Villalpando et al., (2001) argumentan que la prueba de Tukey es usado en conjunto con ANOVA y ayuda a determinar la correlación en base a las medias entre variables, este test faculta a no definir un solo resultante, sino a especificar entre que variables existe diferencia o similitud estadística; el valor analizado por la tabla de Tukey usa como numerador al número de eventos y denominador a los grados de libertad del error; es usado cuando los valores muestrales son iguales, lo cual adjudica un nivel de significancia favorable. La ecuación para valorar esta metodología es:

$$
w=q x \sqrt{\frac{C M E}{r}}
$$

\section{Donde:}

- $q=$ valorado por el número de tratamiento y los grados de libertad del error

- $C M E=$ cuadrado del medio del error

- $r=$ número de repeticiones

\section{Resultados y Discusión}

Con la finalidad de estratificar y segmentar de forma correcta los datos se observará la información en base a rangos, el planteamiento se hará iniciando en el primer cuartil (25\%) finalizando en el tercer cuartil (75\%), con esta formulación se suprimen los datos atípicos, 
brindando confianza en la evaluación estadística; los rangos parametrizados por cuartiles son los siguientes:

Tabla 2

Cuartiles de los indicadores de productividad

\begin{tabular}{ccccccc}
\hline $\begin{array}{c}\text { Cua } \\
\text { rtil }\end{array}$ & $\begin{array}{c}\text { Índice } \\
\text { Dupont }\end{array}$ & $\begin{array}{c}\text { Margen de rentabilidad } \\
\text { neta en ventas }\end{array}$ & $\begin{array}{c}\text { Rotación de } \\
\text { activos }\end{array}$ & $\begin{array}{c}\text { Multiplicador } \\
\text { de capital }\end{array}$ & $\begin{array}{c}\text { Costo del } \\
\text { financiamiento }\end{array}$ & $\begin{array}{c}\text { Costos } \\
\text { operativos }\end{array}$ \\
\hline $\mathbf{2 5 \%}$ & $6,05 \%$ & $0,83 \%$ & 1,075 & 1,86 & $0,00 \%$ & $50,79 \%$ \\
$\mathbf{5 0 \%}$ & $15,83 \%$ & $2,32 \%$ & 1,727 & 3,14 & $0,00 \%$ & $69,19 \%$ \\
$\mathbf{7 5 \%}$ & $37,01 \%$ & $4,89 \%$ & 3,041 & 6,53 & $13,86 \%$ & $82,91 \%$ \\
\hline
\end{tabular}

Fuente: Elaboración propia, mediante la representación visual en diagrama de caja simple SPSS v.21

Tabla 3

Diagramas de los indicadores

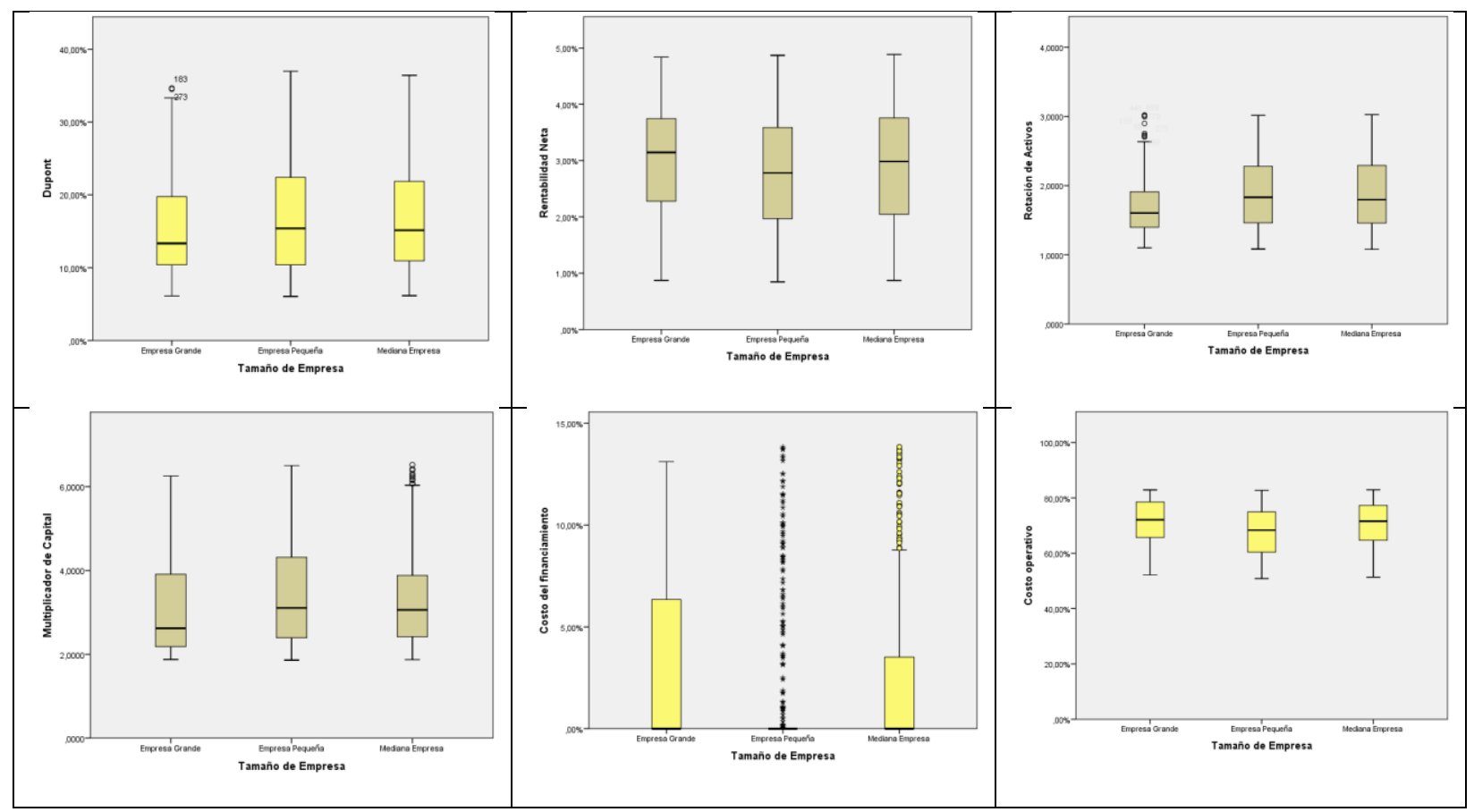

Fuente: Elaboración propia, mediante la representación visual en diagrama de caja simple SPSS v.21

Mediante la observación de los datos se puede enfatizar que el potencial de las empresas pertenecientes a la actividad económica $G 46$ es la rotación de los activos y el multiplicador de capital, mientras que la rentabilidad neta es un valor relativamente bajo; por otra parte, los gráficos permiten aludir que la distribución de la diferencia entre medias pareciera no ser tan significativa, sin embargo, el estadístico enfatizará en dicha aseveración. 


\section{Tabla 4}

Hausman

\begin{tabular}{lcccc}
\hline \multicolumn{1}{c}{ Variable } & $(b)$ & $(B)$ & $(b-B)$ & $s q r t$ \\
\cline { 2 - 5 } & Efectos Fijos & Efectos Aleatorios & Diferencia & S.E. \\
\hline Margen de rentabilidad sobre las ventas & 6,147463 & 6,148833 & $-0,001370$ & 0,007969 \\
Rotación de activos & 0,084860 & 0,084897 & $-0,000037$ & 0,000098 \\
Multiplicador de capital & 0,046214 & 0,046220 & $-0,000006$ & 0,000011 \\
Costo del financiamiento & 0,010040 & 0,009306 & 0,000734 & 0,002097 \\
Costo Operativo & $-0,001435$ & $-0,001439$ & 0,000004 & 0,000634 \\
Empresa pequeña & 0,004909 & 0,004952 & $-0,000043$ & 0,000132 \\
Mediana empresa & 0,002197 & 0,002225 & $-0,000028$ & 0,000149
\end{tabular}

\section{Prob $>$ Chi $^{2}=0,9798$}

Fuente: Elaboración propia en base a los datos de las empresas, representando Hausman, sigmamore con el estadístico STATA v.16

Una vez ejemplificado los datos y con un $P r o b>C h i^{2}$ superior a 0,9798 se determina que el modelo de efectos aleatorios es eficiente para el tratamiento de datos; el modelo se presenta a continuación:

\section{Tabla 5}

Efectos aleatorios

\begin{tabular}{|c|c|c|c|c|}
\hline Source & & SS & df & MS \\
\hline Model & & 5,14 & 7 & 0,73 \\
\hline Residual & & 0,48 & 937 & 0 \\
\hline Total & & 5,63 & 944 & 0,01 \\
\hline Sujetos & & $=$ & \multicolumn{2}{|c|}{945} \\
\hline Prob $>F$ & & $=$ & \multicolumn{2}{|c|}{0,000} \\
\hline$R^{2}$ & & $=$ & \multicolumn{2}{|c|}{0,9144} \\
\hline Índice Dupont & Coef. & Std, Err, & Valor (t) & $\mathbf{P}>\mathbf{t}$ \\
\hline Margen de rentabilidad sobre las ventas & 6,1488 & 0,0769 & 79,97 & 0,000 \\
\hline Rotación de activos & 0,0849 & 0,0015 & 55,74 & 0,000 \\
\hline Multiplicador de capital & 0,0462 & 0,0006 & 72,23 & 0,000 \\
\hline Costo del financiamiento & 0,0093 & 0,0204 & 0,46 & 0,649 \\
\hline Costo Operativo & $-0,001$ & 0,0087 & $-0,17$ & 0,869 \\
\hline Empresa pequeña & 0,005 & 0,0023 & 2,16 & 0,031 \\
\hline Mediana empresa & 0,0022 & 0,0023 & 0,97 & 0,331 \\
\hline Constante & $-0,3245$ & 0,0081 & $-39,88$ & 0 \\
\hline
\end{tabular}

Fuente: Elaboración propia en base a los datos de las empresas, representando xtreg, re con el estadístico STATA v.16

Mediante el análisis de efectos aleatorios con un nivel de significancia de 0,000, es decir, con un nivel de confianza del 99,99\% ejemplifica que el modelo es veraz, asimismo, el modelo 
expuesto y sus variables adjudican una explicación del 91,44\%, previa observación se determina que, el margen de rentabilidad sobre ventas (+), rotación de activos $(+)$, multiplicador de capital $(+)$ y la empresa categorizada como pequeña (+) posee una asociatividad y relación mayor al $95 \%$ del nivel de confianza, sin embargo, el modelo muestra que, el financiamiento y el costo operativo no son variables influyentes.

\section{Tabla 6}

Efectos aleatorios

\begin{tabular}{ccccccc}
\hline & $\begin{array}{c}\text { Índice } \\
\text { Dupont }\end{array}$ & $\begin{array}{c}\text { Margen de } \\
\text { rentabilidad } \\
\text { sobre las } \\
\text { ventas }\end{array}$ & $\begin{array}{c}\text { Rotación } \\
\text { de activos }\end{array}$ & $\begin{array}{c}\text { Multiplicador } \\
\text { de capital }\end{array}$ & $\begin{array}{c}\text { Costo del } \\
\text { financiamiento }\end{array}$ & $\begin{array}{c}\text { Costo } \\
\text { Operativo }\end{array}$ \\
\hline $\begin{array}{c}\text { Índice Dupont } \\
\text { Margen de }\end{array}$ & 1 & & & & \\
$\begin{array}{c}\text { rentabilidad } \\
\text { sobre las ventas } \\
\text { Rotación de }\end{array}$ & $0,5172^{*}$ & 1 & & & \\
$\begin{array}{c}\text { activos } \\
\text { Multiplicador }\end{array}$ & $0,2827^{*}$ & $-0,2163^{*}$ & 1 & & \\
$\begin{array}{c}\text { de capital } \\
\text { Costo del }\end{array}$ & $0,4523^{*}$ & $-0,2419^{*}$ & $-0,1460^{*}$ & 1 & 1 & \\
financiamiento & $-0,0257$ & 0,0444 & $-0,0428$ & $-0,0517$ & & \\
$\begin{array}{c}\text { Costo } \\
\text { Operativo }\end{array}$ & $-0,0094$ & $-0,0531$ & $0,0837 *$ & $-0,0099$ & $-0,0213$ & 1 \\
\hline
\end{tabular}

Fuente: Elaboración propia en base a los datos de las empresas, representando pwcorr, star (95\%) con el estadístico STATA v.16

Con la finalidad de observar la relación entre variables de carácter individual, mediante correlación se determina que: el margen de rentabilidad sobre las ventas, rotación de activos, multiplicador de capital son variables con significancia estadística del 95\% y con una relación proporcional positiva con relación al índice Dupont; rotación de activos, multiplicador de capital se presentan como variables significativas con relación inversa ante el margen de rentabilidad sobre las ventas; por último, el multiplicador de capital se posiciona como variable significativa con una relación inversamente proporcional con respecto a la rotación de los activos.

\section{Tabla 7}

Descriptivos

\begin{tabular}{|c|c|c|c|c|c|c|c|c|c|}
\hline & & \multirow{2}{*}{$\mathbf{N}$} & \multirow{2}{*}{ Media } & \multirow{2}{*}{$\begin{array}{l}\text { Desviación } \\
\text { típica }\end{array}$} & \multirow{2}{*}{$\begin{array}{l}\text { Error } \\
\text { típico }\end{array}$} & \multicolumn{2}{|c|}{$\begin{array}{l}\text { Intervalo de confianza } \\
\text { para la media al } 95 \%\end{array}$} & \multirow{2}{*}{ Mínimo } & \multirow{2}{*}{ Máximo } \\
\hline & & & & & & $\begin{array}{l}\text { Limite } \\
\text { inferior }\end{array}$ & $\begin{array}{l}\text { Limite } \\
\text { superior }\end{array}$ & & \\
\hline \multirow{2}{*}{$\begin{array}{c}\text { Costo del } \\
\text { financiamiento }\end{array}$} & $\begin{array}{l}\text { Empresa } \\
\text { Grande }\end{array}$ & 139 & $3,25 \%$ & $4,11 \%$ & $0,35 \%$ & $2,56 \%$ & $3,94 \%$ & $0,00 \%$ & $13,11 \%$ \\
\hline & $\begin{array}{l}\text { Empresa } \\
\text { Mediana }\end{array}$ & 361 & $2,44 \%$ & $3,89 \%$ & $0,20 \%$ & $2,04 \%$ & $2,85 \%$ & $0,00 \%$ & $13,84 \%$ \\
\hline
\end{tabular}




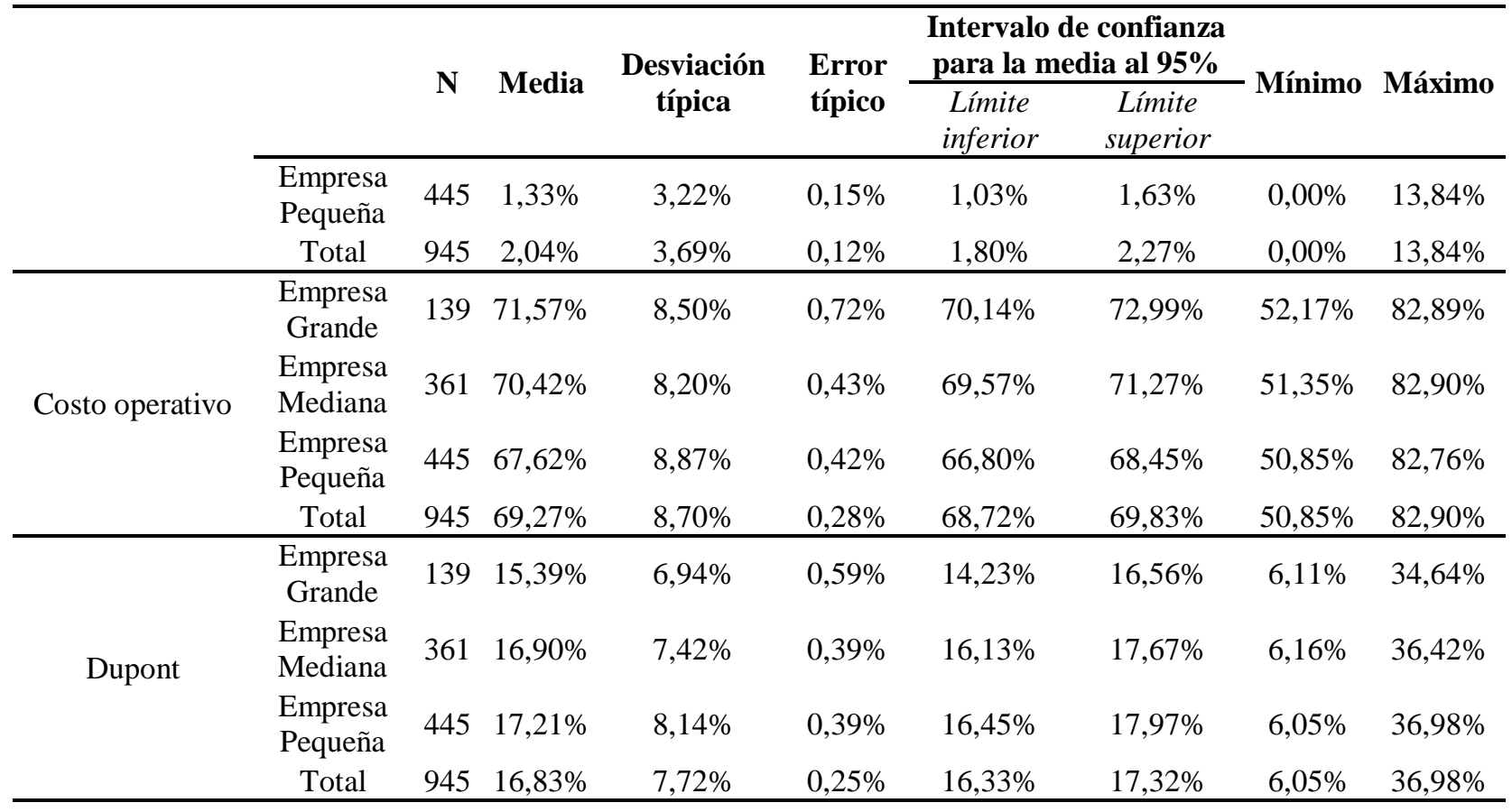

Tabla 8

ANOVA de un factor

\begin{tabular}{|c|c|c|c|c|c|c|}
\hline & & $\begin{array}{l}\text { Suma de } \\
\text { cuadrados }\end{array}$ & gl & $\begin{array}{c}\text { Media } \\
\text { cuadrática }\end{array}$ & $\mathbf{F}$ & Sig. \\
\hline \multirow{3}{*}{$\begin{array}{c}\text { Costo del } \\
\text { financiamiento }\end{array}$} & Inter-grupos & 488,352 & 2 & 244,176 & 18,565 & ,000 \\
\hline & Intra-grupos & 12389,596 & 942 & 13,152 & & \\
\hline & Total & 12877,948 & 944 & & & \\
\hline \multirow{3}{*}{$\begin{array}{c}\text { Costo } \\
\text { operativo }\end{array}$} & Inter-grupos & 2416,335 & 2 & 1208,167 & 16,471 & $\overline{, 000}$ \\
\hline & Intra-grupos & 69096,496 & 942 & 73,351 & & \\
\hline & Total & 71512,831 & 944 & & & \\
\hline \multirow{3}{*}{ Dupont } & Inter-grupos & 354,070 & 2 & 177,035 & 2,983 & ,051 \\
\hline & Intra-grupos & 55904,773 & 942 & 59,347 & & \\
\hline & Total & 56258,843 & 944 & & & \\
\hline
\end{tabular}

Fuente: Elaboración propia en base a los datos de las empresas, representando ANOVA de un factor con el estadístico SPSS v.21

El Tratamiento de la información con respecto a ANOVA de un factor determina valores con alta significancia mayor al 99,99\% del nivel de confianza en el costo de financiamiento y operativo, lo cual asevera que, entre los tamaños de empresas adjudican valores diferentes en estas categorías, por otro lado, en el índice Dupont se presenta una significancia de 0,051, lo cual indica que en cierta proporción es algo explicativo con respecto al modelo analizado, sin embargo, determinamos que el índice es similar entre los grupos analizados, para solventar dicha característica de congruencia al valor $\mathrm{P}$, se hará continuidad con el test de Turkey. 


\section{Tabla 9}

Tabla de Turkey

\begin{tabular}{|c|c|c|c|c|c|c|c|}
\hline \multirow{2}{*}{$\begin{array}{c}\text { Variable } \\
\text { dependiente }\end{array}$} & \multirow{2}{*}{ (I) $\mathrm{xe}$} & \multirow{2}{*}{ (J) $\mathrm{xe}$} & \multirow{2}{*}{$\begin{array}{c}\text { Diferencia de } \\
\text { medias (I-J) }\end{array}$} & \multirow{2}{*}{ Error típico } & \multirow{2}{*}{ Sig. } & \multicolumn{2}{|c|}{$\begin{array}{c}\text { Intervalo de confianza al } \\
95 \% \\
\end{array}$} \\
\hline & & & & & & $\begin{array}{l}\text { Límite } \\
\text { inferior }\end{array}$ & $\begin{array}{c}\text { Límite } \\
\text { superior }\end{array}$ \\
\hline \multirow{8}{*}{$\begin{array}{l}\text { Costo del } \\
\text { financiamiento }\end{array}$} & \multirow{3}{*}{$\begin{array}{c}\text { Empresa } \\
\text { Grande }\end{array}$} & Empresa & $0,80825 \%$ & $0,36202 \%$ & ,066 & $-0,0416 \%$ & $1,6581 \%$ \\
\hline & & Mediana & & & & & \\
\hline & & Empresa & $1,92358 \% *$ & $0,35239 \%$ &, 000 & $1,0964 \%$ & $2,7508 \%$ \\
\hline & & Empresa & $-0.80825 \%$ & $0,36202 \%$ & 066 & $-1.6581 \%$ & $00416 \%$ \\
\hline & Empresa & Grande & $-0,0002010$ & 0,2020270 &, 000 & $-1,050170$ & 0,04107 \\
\hline & Mediana & $\begin{array}{c}\text { Empresa } \\
\text { Pequeña }\end{array}$ & $1,11533 \% *$ & $0,25688 \%$ &, 000 & $0,5123 \%$ & $1,7183 \%$ \\
\hline & Empresa & $\begin{array}{c}\text { Empresa } \\
\text { Grande }\end{array}$ & $-1,92358 \% *$ & $0,35239 \%$ & ,000 & $-2,7508 \%$ & $-1,0964 \%$ \\
\hline & Pequeña & $\begin{array}{l}\text { Empresa } \\
\text { Mediana }\end{array}$ & $-1,11533 \% *$ & $0,25688 \%$ &, 000 & $-1,7183 \%$ & $-0,5123 \%$ \\
\hline \multirow{8}{*}{ Costo operativo } & \multirow{3}{*}{$\begin{array}{c}\text { Empresa } \\
\text { Grande }\end{array}$} & Empresa & $1,14498 \%$ & $0,85492 \%$ & ,374 & $-0,8619 \%$ & $3,1518 \%$ \\
\hline & & Mediana & & & & & \\
\hline & & $\begin{array}{l}\text { Empresa } \\
\text { Pequeña }\end{array}$ & $3,94177 \% *$ & $0,83219 \%$ &, 000 & $1,9883 \%$ & $5,8953 \%$ \\
\hline & \multirow{3}{*}{$\begin{array}{l}\text { Empresa } \\
\text { Mediana }\end{array}$} & Empresa & $-1,14498 \%$ & $0,85492 \%$ & ,374 & $-3,1518 \%$ & $0,8619 \%$ \\
\hline & & Empresa & $2.79680 \% *$ & $0.60665 \%$ & 000 & $13727 \%$ & $42209 \%$ \\
\hline & & Pequeña & & & & & \\
\hline & \multirow{2}{*}{$\begin{array}{l}\text { Empresa } \\
\text { Pequeña }\end{array}$} & $\begin{array}{c}\text { Empresa } \\
\text { Grande }\end{array}$ & $-3,94177 \% *$ & $0,83219 \%$ &, 000 & $-5,8953 \%$ & $-1,9883 \%$ \\
\hline & & $\begin{array}{l}\text { Empresa } \\
\text { Mediana }\end{array}$ & $-2,79680 \% *$ & $0,60665 \%$ &, 000 & $-4,2209 \%$ & $-1,3727 \%$ \\
\hline \multirow{12}{*}{ Dupont } & \multirow{4}{*}{$\begin{array}{c}\text { Empresa } \\
\text { Grande }\end{array}$} & Empresa & $-1,50892 \%$ & $0,76899 \%$ &, 122 & $-3,3141 \%$ & $0,2962 \%$ \\
\hline & & Mediana & & & & & \\
\hline & & Empresa & $-1,81973 \% *$ & $0,74855 \%$ &, 040 & $-3,5769 \%$ & $-0,0626 \%$ \\
\hline & & Pequeña & & & & & \\
\hline & \multirow{4}{*}{$\begin{array}{l}\text { Empresa } \\
\text { Mediana }\end{array}$} & Empresa & $1,50892 \%$ & $0,76899 \%$ &, 122 & $-0,2962 \%$ & $3,3141 \%$ \\
\hline & & Grande & & & & & \\
\hline & & Empresa & $-0,31081 \%$ & $0,54567 \%$ & ,836 & $-1,5917 \%$ & $0,9701 \%$ \\
\hline & & Pequeña & & & & & \\
\hline & \multirow{4}{*}{$\begin{array}{l}\text { Empresa } \\
\text { Pequeña }\end{array}$} & Empresa & $1,81973 \% *$ & $0,74855 \%$ &, 040 & $0,0626 \%$ & $3,5769 \%$ \\
\hline & & Grande & & & & & \\
\hline & & Empresa & $0,31081 \%$ & $0,54567 \%$ & ,836 & $-0,9701 \%$ & $1,5917 \%$ \\
\hline & & Mediana & & & & & \\
\hline
\end{tabular}

*. La diferencia de medias es significativa al nivel 0.05 .

Fuente: Elaboración propia en base a los datos de las empresas, representando la prueba de Turkey (Post Hoc) con el estadístico SPSS v.21

Turkey determina y converge los datos de forma exitosa y concluye que el costo del financiamiento entre la empresa grande con respecto a la mediana presenta una ligera diferencia entre medias de $0,80825 \%$ con un nivel de significancia de 0,066 asevera que son iguales entre sí; por otro lado, la empresa grande con respecto a la pequeña presenta una diferencia entre medias 
de $1,92358 \%$ con un nivel de significancia de 0,000 ratifica que existe diferencia explicativa, la empresa pequeña con respecto a la mediana presenta una diferencia entre medias de $-1,11533 \%$ y un nivel de significancia de 0,000 lo cual determina que son diferentes entre sí.

Asimismo, en la estratificación de costo operativo la empresa grande con relación a la empresa mediana presenta diferencias entre medias de 1,14498\% con un nivel de significancia de 0,374 lo cual determina que son relativamente congruentes entre sí, la empresa grande con respecto a la pequeña presenta una diferencia entre grupos de $3,94177 \%$ con un nivel de significancia de 0,00 lo cual indica que existe una diferencia significativa entre estas categorías, la empresa pequeña con respecto a la mediana presenta una diferencia entre medias de $-2,79680 \%$ y un nivel de significancia de 0,000 lo cual determina que son diferentes entre sí.

Por último, el índice Dupont en relación a la empresa grande con respecto a la mediana adjudica una diferencia entre grupos de $0,76899 \%$ con un nivel de significancia de 0,122 asevera que son similares entre sí, la empresa grande con respecto a la pequeña adjudica una diferencia entre medias de $-1,82973 \%$ y con un nivel de significancia de 0,040 determina que son disimiles entre sí, la empresa pequeña con respecto a la mediana presenta una diferencia entre medias de $0,31081 \%$ y un nivel de significancia de 0,836 lo cual determina que son muy similares entre sí.

\section{Conclusiones}

El desarrollo del estudio tuvo aseveraciones disímiles a la de Vilabella y Silvosa (2001), la cual menciona que el tamaño no influye en el estrato financiero de las empresas, sin embargo, los resultados determinaron que en el costo financiero y operativo en las empresas grandes con las medianas son similares, sin embargo, la pequeña presenta resultados diferentes, mediante la estratificación de los datos se determina que las empresas pequeñas tienen menos costo financiero, menos costos operativos y un índice Dupont más elevado, lo cual es beneficioso e implica un preámbulo a la excelencia administrativa, por otro lado, las premisas teóricas enfatizan que, mientras más carga de capital y recursos, es necesario una administración compleja, afirman que el tratamiento y manejo de infraestructura, talento humano y procesos productivos aumentan la dificultad en la consecución de rendimiento, pero, en un enfoque al mercado tienen más beneficios.

Basándonos en el costo financiero es importante disputar con lo argumentado por Jiménez Naharro y Palacín Sánchez (2007), afirma que el endeudamiento de las empresas circunda el 70\%, pero, las empresas pequeñas y medianas no tienen un acceso tan rápido y eficiente como las empresas grandes a recursos externos, uno de los problemas que se presenta en esta afirmación es el costo financiero, en Ecuador los créditos con Instituciones Financieras Intermediarias (IFis) presentan intereses sumamente altos, que en ciertos casos se encuentran superiores al 25\% (microcrédito) según los datos del Banco Central del Ecuador, Sanz y Ayca (2006) mencionan que el costo financiero es uno de los principales problemas de insolvencia en las empresas Latinoamericanas, debido a su alto valor que es imposible cubrir con la operatividad; por otro lado, argumentan que si no existe esa captación monetaria es complicado acceder a un mercado competitivo, debido a esto, es propicio que las empresas consideren las distintas fuentes de financiamiento existentes en el entorno, con el objetivo de controlar el riesgo de liquidez y solvencia. Aunque las particularidades de las empresas abunden, es propicio forjar ideales 
administrativos alineados a la generación de valor económico, es necesario que el talento humano encargado de la dirección esté en constante indagación del área desempeñada para tomar las mejores decisiones, Colina (2006) afirma que los costos no son aberrantes, estos permiten tomar decisiones correctivas y de mejora continua en la organización.

Mediante los estadísticos que buscaban determinar variables significativas en la productividad (Dupont) se determinó que tanto el costo financiero como operativo no son valores significativos, por otro lado, mediante categóricos se comprueba que pertenecer a una pequeña empresa influye de forma positiva en la consecución de productividad, por medio de la correlación, se esclarece que el estudio difiere a lo indagado por Cabellos Mendo y Naito Endo (2015), el cual asegura que el índice Dupont tiene alta relación con el costo de la deuda.

Aunque la información apunta que la empresa pequeña es más productiva es necesario observar y plantear incidencias que permitan categorizar diferentes cuestiones con respecto a esta entidad, los futuros trabajos, deberán ligarse a la liquidez, eficiencia técnica, eficacia en la consecución de beneficios y a la gestión.

\section{Referencias Bibliográficas}

Acín, J. A. M. (1996). Estructura financiera y crecimiento de las PYMES. Economía industrial, 310, 29-40. https://dialnet.unirioja.es/servlet/articulo?codigo=139890

Aguiar-Díaz, I., \& Díaz-Díaz, N. L. (2015). Audit quality, Second-Tier and size: Effect on the Spanish private distressed firms. Spanish Journal of Finance and Accounting, 44(1), 2446. https://doi.org/10.1080/02102412.2014.982386

Aguilar, L. J. (2016). Big Data, Análisis de grandes volúmenes de datos en organizaciones. Alfaomega Grupo Editor.

Altman, E. I. (1968). Financial Ratios, Discriminant Analysis and the Prediction of Corporate Bankruptcy. The Journal of Finance, 23(4), 589-609. https://doi.org/10.1111/j.15406261.1968.tb00843.x

Brynjolfsson, E., \& Yang, S. (1996). Information technology and productivity: A review of the literature. Advances in computers, 43, 179-214.

Cabellos Mendo, J., y Naito Endo, M. (2015). Determinantes de la rentabilidad de las instituciones microfinancieras peruanas en el periodo 2006-2013: Un enfoque según el modelo de Dupont y la teoría de Modigliani-Miller. Repositorio de la Universidad del Pacífico - UP. http://repositorio.up.edu.pe/handle/11354/1697

Camison, C., Lapiedra, R., Segarra, M., y Boronat, M. (2002). Meta-Análisis De La Relación Entre Tamaño De Empresa E Innovación. Working papers = Documentos de trabajo: Serie EC, No. 15, 200253 págs.

Colina, M. A. V. (2006). Gerencia basada en valor: La inclusión del costo financiero como un costo de oportunidad. Actualidad contable FACES, 9(13), 154-165.

Correa-García, J. A., Gómez Restrepo, S., y Londoño Castañeda, F. (2018). Indicadores financieros y su eficiencia en la explicación de la generación de valor en el sector cooperativo. Revista Facultad de Ciencias Económicas, 26(2), 129-144. https://doi.org/10.18359/rfce.3859 
Cuadros, Q., y Ángel, M. (2019). Implementación de la Metodología PHVA para incrementar la productividad en una empresa de servicios. Repositorio de Tesis - UNMSM. https://cybertesis.unmsm.edu.pe/handle/20.500.12672/10822

De la Peña Gutiérrez, A. (2014). Auditoría. Un enfoque práctico. Editorial Paraninfo.

Deming, W. E., y Medina, J. N. (1989). Calidad, productividad y competitividad: La salida de la crisis. Ediciones Díaz de Santos.

Dewan, S., \& Kraemer, K. L. (2000). Information technology and productivity: Evidence from country-level data. Management science, 46(4), 548-562.

Díaz, I. A. (2012). Finanzas Corporativas en la Práctica. Delta Publicaciones.

Diez, D., Çetinkaya-Rundel, M., \& Barr, C. (2019). Open Intro Statistics: Fourth Edition.

García, A. (1981). Clasificación industrial de todas las actividades económicas: CIIU. Ministerio de Trabajo.

García, O. L. (2009). Administración financiera fundamentos y aplicaciones. Prensa Moderna.

García-Villalpando, J. A., Castillo-Morales, A., Ramírez-Guzmán, M. E., Rendón-Sánchez, G., y Larqué-Saavedra, M. U. (2001). Comparación de los procedimientos de Tukey, Duncan, Dunnett, Hsu y Bechhofer para selección de medias. Agrociencia, 35(1), 79-86.

Garrido, C., y Peres Núñez, W. (1998). Las grandes empresas y grupos industriales latinoamericanos en los años noventa. https://repositorio.cepal.org//handle/11362/31047

Girden, E. R. (1992). ANOVA: Repeated Measures. SAGE.

Gitman, L. J. (2003). Principios de administración financiera. Pearson Educación.

Granados, R. M. (2011). Efectos fijos o aleatorios: Test de especificación. 5.

Gutiérrez Pulido, H. (2010). Calidad total y productividad. McGraw Hill Educación. http://uprid2.up.ac.pa:8080/xmlui/handle/123456789/1392

Haro, A. F. (2021). La estructura financiera y el fracaso empresarial: Una apreciación a las grandes empresas de pesca y acuicultura. Revista Ciencia Multidisciplinaria CUNORI, 5(1), 1-16. https://doi.org/10.36314/cunori.v5i1.148

Herrera, J. L. (2012). Productividad. Palibrio.

Ishikawa, K. (2003). ¿Qué es el control total de calidad? Editorial Norma.

Jiménez Naharro, F., y Palacín Sánchez, M. J. (2007). Determinantes de la estructura financiera de la empresa. https://idus.us.es/handle/11441/43891

Luna, Y. B. (2015). Auditoría integral: Normas y procedimientos. Ecoe Ediciones.

López-Sánchez, J. I. (2004). ¿Pueden las tecnologías de la información mejorar la productividad? Universia Business Review, (1), 82-95. https://www.redalyc.org/articulo.oa?id=43300108

Malvicino, F., y Yoguel, G. (2017). Big data: Avances recientes a nivel internacional y perspectivas para el desarrollo local. CIECTI.

Martínez, Y. A., Alfonso, B. B., y Marichal, L. L. (2012). Auditoría con Informática a Sistemas Contables. Revista Arquitectura e Ingeniería, 6(2), 4-14. https://dialnet.unirioja.es/servlet/articulo?codigo $=4004937$

Masgrau, E. G. (2005). El apalancamiento financiero: De cómo un aumento del endeudamiento puede mejorar la rentabilidad financiera de una empresa. Revista de contabilidad y dirección, 2, 71-91. https://dialnet.unirioja.es/servlet/articulo?codigo=2235220

Montero, R. (2005). Test de Hausman. Documentos de Trabajo en Economía Aplicada, 3. https://www.ugr.es/ montero/matematicas/hausman.pdf

Morillo, M. (2001). Rentabilidad financiera y reducción de costos. Actualidad Contable FACES, 4(4), 35-48. http://www.redalyc.org/articulo.oa?id=25700404\&iCveNum=676 
Mutl, J., \& Pfaffermayr, M. (2011). The Hausman test in a Cliff and Ord panel model. The Econometrics Journal, 14(1), 48-76. https://doi.org/10.1111/j.1368-423X.2010.00325.x

Pelazas, A. (2015). Planificación de la auditoría. Ediciones Paraninfo, S.A.

Prats, M., y Merino, F. (2015). La importancia del tamaño en la empresa española. ICE Revista de Economía, 885, 13.

Restrepo, L. F., \& González, J. (2007). From pearson to Spearman. Revista Colombiana de Ciencias Pecuarias, 20(2), 183-192.

Rivas Martínez, S. P., y Montero Cabas, I. A. (2020). Análisis y desarrollo de estrategias para la optimización de los indicadores financieros de la firma Scain administración e ingeniería SAS. [Thesis, Corporación Universitaria Minuto de Dios]. En Reponame: Digitales Uniminuto. https://repository.uniminuto.edu/handle/10656/10209

Rosillón, M. A. N. (2009). Análisis financiero: Una herramienta clave para una gestión financiera eficiente. Revista venezolana de Gerencia, 14(48), 606-628. http://ve.scielo.org/scielo.php?script=sci_arttext\&pid=S131599842009000400009\&lng=es\&tlng=es.

Rubio-Hurtado, M.-J., y Berlanga-Silvente, V. (2012). Cómo aplicar las pruebas paramétricas bivariadas $\mathrm{t}$ de Student y ANOVA en SPSS. Caso práctico. https://core.ac.uk/display/39101713

Sanz, L. J., y Ayca, J. (2006). Costo (financiero) de problemas de insolvencia en América Latina. Un caso de estudio. Academia. Revista Latinoamericana de Administración, 36, 65-81.

Scaramussa, S. A., Ribeiro, A. A., y Reisdorfer, V. K. (2010). La contribución del Balanced Scorecard como instrumento de gestión estratégica en el apoyo a la gerencia. Visión de futuro, 13(1), 95-109. https://dialnet.unirioja.es/servlet/articulo?codigo=7408098

Stihle, L., \& Wold, S. (1989). Analysis of variance (ANOVA). Chemometrics and Intelligent Laboratory Systems, 6(4), 259-272. https://doi.org/10.1016/0169-7439(89)80095-4

SUPERCIAS. (2020). RankingCias. Empresas sujetas al control de la Superintendencia de Compañías, Valores y Seguros. https://appscvs.supercias.gob.ec/rankingCias/

Tárraga, A. J. (2011). Uso de indicadores financieros para evaluar el impacto de las Prácticas de $\begin{array}{llll}\text { Alta } & \text { Implicación. } & \text { 32-43. }\end{array}$ https://dialnet.unirioja.es/servlet/articulo?codigo $=4787171$

Tascón, M. (2013). Introducción: Big Data. Pasado, presente y futuro. Telos: Cuadernos de $\begin{array}{llll}\text { comunicación } & e & \text { innovación, } & \text { 47-50. }\end{array}$ https://dialnet.unirioja.es/servlet/articulo?codigo $=4423775$

Terreno, D. D. (2013). Analisis empírico de la relación de Dupont. Contabilidad y Decisiones, 5, 11-11.

Torrecilla, O. D. (2005). Clima organizacional y su relación con la productividad laboral. Mza., documento de cátedra, Facultad de Ciencias Políticas y Sociales, UNC.

Vilabella, L. B., y Silvosa, A. R. C. (2001). Incidencia del tamaño sobre el comportamiento financiero de la empresa. Un análisis empírico con pymes gallegas. 10, 24.

Zabaleta García, H. E. (2012). Análisis de rentabilidad de la Distribuidora la Princesa: Roa Roe/análisis Dupont y simulaciones - flujo de caja 2009-2011. https://repositorio.utb.edu.co/handle/20.500.12585/953

Zapata, A. (2016). Ciclo de la calidad PHVA. Universidad Nacional de Colombia. 